\title{
Major Inflammatory Markers and Their Significance in Predicting Severity of COVID-19 Disease Pattern
}

\author{
Abdullah I. Aedh ${ }^{1,2^{*}}$; Ali Hadi Al Hajri ${ }^{3}$; Abdulaziz S. Alshahrani ${ }^{1,2}$; Mohammed A. Adam ${ }^{3}$; \\ Abubaker Alsiddig Dahab ${ }^{3}$; Asaad M. A. Babker ${ }^{4}$; Hatem Mohamed ${ }^{1}$ \\ ${ }^{I}$ College of Medicine, Najran University, Najran, Kingdom of Saudi Arabia \\ ${ }^{2}$ Najran University Hospital, Najran, Kingdom of Saudi Arabia \\ ${ }^{3}$ Najran Armed Forces Hospital, Najran, Kingdom of Saudi Arabia \\ ${ }^{4}$ College of Health Sciences, Gulf Medical University, Ajman, United Arab Emirates
}

\begin{abstract}
The unanticipated outbreak of the COVID-19 pandemic has shocked the world in terms of both lives and livelihood. SARS$\mathrm{CoV}-2$ virus primarily affects the respiratory system, although other organ systems are also involved. Early diagnosis followed up by a retrospective analysis and tracking of a few markers relevant to the immunological status of the individual may aid in determining the state of the patient's disease prognosis. The aim of the present study was to evaluate immunological parameters such as neutrophil to lymphocyte ratio (NLR), C-reactive protein (CRP), and D-dimer, taking into account the patient's age and oxygen saturation level.

Our retrospective analysis of clinical data revealed that such parameters as CRP, D-dimer, and NLR should be taken into consideration to predict severe COVID-19-related complications. The data obtained indicate that patients over age 60 are especially vulnerable to severe COVID-19.(International Journal of Biomedicine. 2021;11(4):488-492.)
\end{abstract}

Key Words: COVID-19 $\bullet$ neutrophilia $・$ D-dimer $\bullet$ C-reactive protein

For citation: Abdullah I. Aedh AI, Al Hajri AH, Alshahrani AS, Adam MA, Dahab AA, Babker AMA, Mohamed H. Major Inflammatory Markers and Their Significance in Predicting Severity of COVID-19 Disease Pattern. International Journal of Biomedicine. 2021;11(4):488-492. doi:10.21103/Article11(4)_OA13

\section{Introduction}

The unanticipated outbreak of the COVID-19 pandemic has shocked the world in terms of both lives and livelihood. The origin of the SARS-CoV-2 virus is itself a debatable mystery; however, its severity is highly unpredictable, as several countries in the world have already experienced serious consecutive waves of infection. As the virus mutates, it becomes more infectious and aggressively hits the host, leading to a higher rate of mortality. ${ }^{(1)}$ Globally, this virus has been considered a major threat in both developed and under-

"Corresponding author: Dr. Abdullah I. Aedh. Associated professor of internal medicine. College of Medicine, Najran University; Najran University Hospital, Najran, Kingdom of Saudi Arabia.E-mail: Dr.abuwaleed3730@hotmail.com developed countries as it mainly halts advancement of the socio-economic status of the country directly.

SARS-CoV-2 establishes residence in the host and multiplies, primarily focusing on the respiratory system and causing a respiratory disease. It enters the epithelial cells of the nasal cavity by engaging the ACE2 receptor with the viral receptor-binding domain (RBD) and begins replicating. ${ }^{(2-4)}$ In the stage of established pulmonary disease, there are viral multiplication and localized inflammation in the lungs that may lead to devastating damage. The most severe stage of the illness manifests as an extrapulmonary, systemic hyperinflammation syndrome. ${ }^{(5)}$

It is highly important to block the viral entry at its initial phase. Therefore, early diagnosis and monitoring are necessary in order to control the disease progression and its clinical complications. ${ }^{(6)}$ Early diagnosis followed by the retrospective analysis and follow-up of few markers could even predict 
the immunological status of the individual. The heightened immunological response and the consecutive transmission of this virus colonizing in the lungs are the two critical factors that determine the severity of the disease. ${ }^{(7)}$

Miriam Merad and Jerome C. Martin highlight that current models of COVID-19 propose three distinct immune stages that are crucial for the ultimate disease course: ${ }^{(8)}$ "In the first stage, early activation of the immune system through the induction of a potent interferon response is important to control the virus. In the second stage, a delayed interferon response may lead to progressive tissue damage. This may ultimately lead to the third stage, a deleterious hyperinflammation characterized by the excessive macrophage activation and coagulation that is seen in patients with severe disease, possibly followed by dysregulation of tissue repair mechanisms and fibrosis."

According to Huertas et al., endothelial cell dysfunction and impaired microcirculatory function contribute markedly to life-threatening complications of COVID-19, such as venous thromboembolic disease and multiple organ involvement. ${ }^{(9)}$ Endothelial dysfunction, complement activation, thrombin generation, platelet and leukocyte recruitment, and the initiation of innate and adaptive immune responses culminate in immunothrombosis, ultimately causing (micro)thrombotic complications, such as deep vein thrombosis, pulmonary embolism, and stroke. In this regard, the activation of coagulation and thrombocytopenia has emerged as a prognostic marker in COVID-19. (10,11) $^{(1)}$

Coagulopathy and D-dimer elevations were seen in $3.75 \%-68.0 \%$ of the COVID-19 patients. ${ }^{(12,13)}$ Yao et al. ${ }^{(14)}$ showed that a D-dimer level of $>2.14 \mathrm{mg} / \mathrm{L}$ predicted inhospital mortality in COVID-19 patients with a sensitivity of $88.2 \%$ and specificity of $71.3 \%$ ( $\mathrm{AUC}=0.85 ; 95 \% \mathrm{CI}=0.77$ 0.92). ${ }^{(14)}$ High levels of CRP have also been used as an indicator of COVID-19 disease severity. Stringer et al. showed that a threshold cut-off of $\mathrm{CRP} \geq 40 \mathrm{mg} / \mathrm{L}$ was associated with mortality in COVID -19 patients. ${ }^{(15)}$

As COVID-19 progresses to the lethal final phase, it is necessary to monitor the surge levels of inflammatory markers in order to prevent the development of cytokine storm and further complications. The aim of the present study was to evaluate immunological parameters such as neutrophil to lymphocyte ratio (NLR), C-reactive protein (CRP), and D-dimer, taking into account the patient's age and oxygen saturation level.

\section{Materials and Methods}

The study was investigated at Najran Armed Forces Hospital. Patients suspected of having COVID-19 were confirmed by qRT-PCR analyzing the fold change expression of SARS-CoV-2 viral-specific genes, such as the $N$ gene and $E$ gene in an oro-nasal throat swab. Strong COVID-19 positive patients with qRT-PCR cycle threshold values of 20-25 consented to enroll in this study. Nearly 121 strong positives volunteered for this clinical investigation. This study included the patients' complete data from the date of admission until the date of discharge or death, information that was available from the hospital administrative records.

We analyzed plasma NLR, CRP, and D-dimer values. All these parameters are closely correlated with immune status and internal microvasculature clotting, which is associated with the lethal phase of the disease. This basic diagnostic profile is usual for the typical multi-specialty hospital.

The case files of the patients were retrospectively reviewed, the required information was extracted, and patients with a co-morbidity were excluded. Treatment with azithromycin, hydroxychloroquine, lopinavir-ritonavir, steroids, or oxygen support was considered as dependent upon the patients' requirements. Asymptomatic patients, were given multivitamins and zinc tablets. The sample size was determined by the time window of the study.

Statistical analysis was performed using the IBM SPSS Statistics for Windows, Version 22.0. Armonk, NY: IBM Corp.).

\section{Results}

A scatter plot (Fig.1) shows a moderate positive linear relationship between NLR and patient's age. The COVID-19 patients above 60 were more sensitive to increased NLR levels. We found a moderate positive linear relationship between CRP and patient's age (Fig.2).

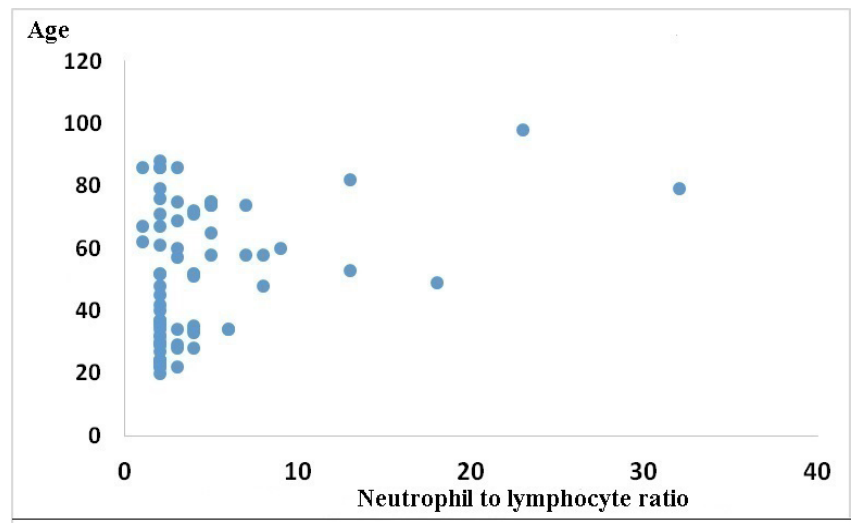

Fig. 1. The relationship between NLR and patient's age $(n=121)$.

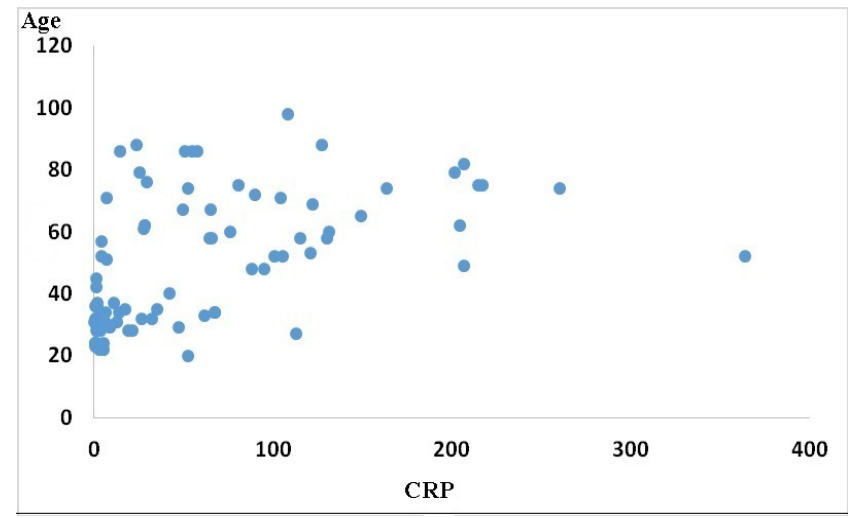

Fig. 2. The relationship between CRP and patient's age $(n=121)$. 
The COVID-19 patients above 60 developed higher CRP values. Figure 3 shows a moderate positive linear relationship between D-dimer and patient's age. The COVID-19 patients above 60 were more sensitive to increased D-dimer values.

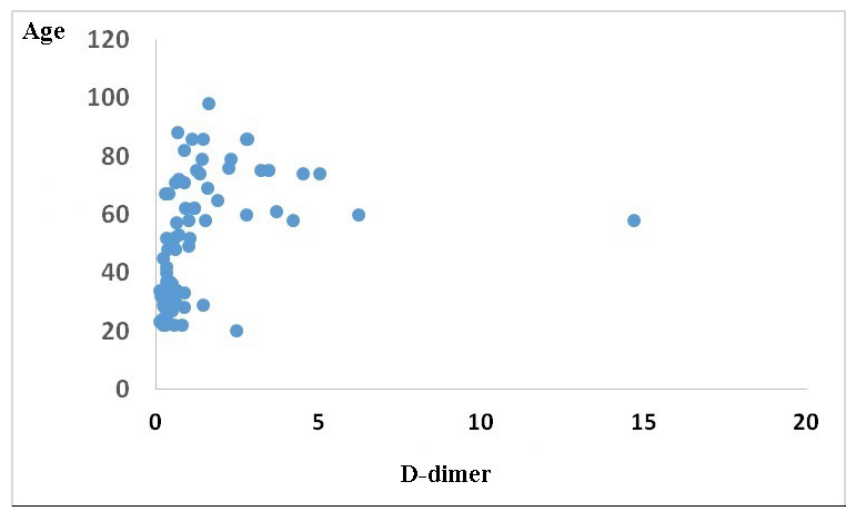

Fig. 3. The relationship between D-dimer and patient's age $(n=121)$.

Figure 4 shows a strong negative linear relationship between patient's age and $\mathrm{SaO}_{2}: \mathrm{SaO}_{2}$ levels decrease in patients above 60 years. In contrast, the age groups ranging between 20-40 and 40-60 maintain consistent $\mathrm{SaO}_{2}$ levels.

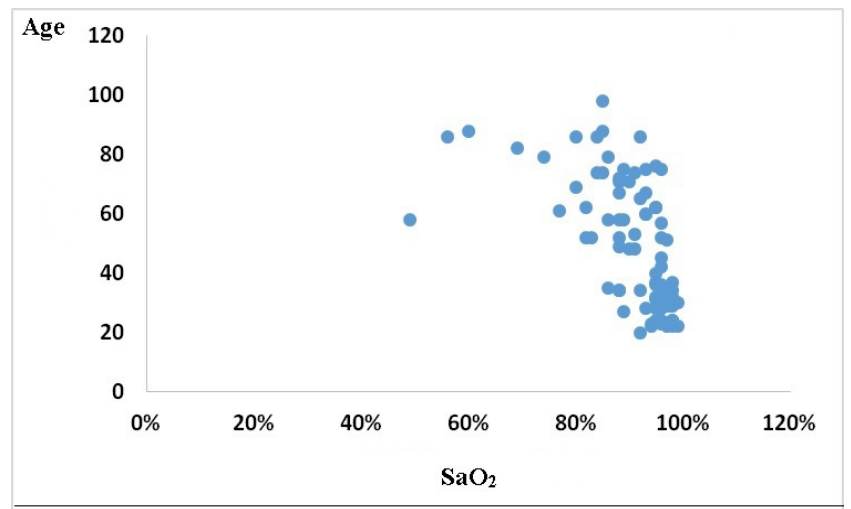

Fig. 4. The relationship between patient's age and $\mathrm{SaO}_{2}$ $(n=121)$.

\section{Discussion}

COVID-19 has quickly spread around the world with high mortality worldwide. The pathological pulmonary damage it has caused may be directly related to the viral destruction of alveolar and bronchial epithelial cells or mass production of proinflammatory cytokines (cytokine storm). ${ }^{(16)}$ In COVID-19, the cytokine storm may result in an uncontrolled systemic inflammatory response, ARDS, multiple organ failure, and death in severe cases. ${ }^{(17,18)}$

Currently, it is clear that hyperinflammation and coagulopathy contribute to disease severity and death in patients infected with SARS-CoV-2 ${ }^{(8)}$ It is believed that higher values of proinflammatory markers are related to extensive lung injury. ${ }^{(19)}$ The neutrophils are known to develop a sophisticated network of extracellular fibers composed of DNA containing histones, called neutrophil extracellular traps (NETs). There is some evidence to suggest that NETosis is conditional on the production of reactive oxygen species. ${ }^{(20)}$

It has been shown that neutrophilia predicts a poor outcome in patients with severe COVID-19 cases, and NLR may be an independent risk factor for the severity of this disease. ${ }^{(21)}$ In a recent article, Shivakumar, with associates from India, found that the neutrophil-to-lymphocyte-to-monocyte ratio and the platelet-to-lymphocyte ratio were significantly prognostic in COVID-19. ${ }^{(22)}$

The high mortality associated with thromboembolic disorders in COVID-19 has prompted clinicians to use D-dimer as a useful marker for assessing the severity of the disease. ${ }^{(23)}$ According to a review by Harvard Medical School researchers, in critically ill patients with COVID-19, elevated levels of D-dimer were found in $100 \%$ of participants, elevated fibrinogen in $74 \%$, and factor V11 in $100 \%{ }^{.24)}$

Nalbant et al. ${ }^{(25)}$ found that the risk of COVID-19 was 20.3 -fold greater when NLR was $\geq 2.4$ in the logistic regression $(P=0.007)$. The authors concluded that NLR is an independent predictor for the diagnosis of COVID-19. In a study by Seyit et al., ${ }^{(26)}$ the $\operatorname{CRP}(P=0.0001)$ and NLR $(P=0.001)$ remained significantly higher in the patients with positive SARS-CoV-2 PCR test results.

The biological changes linked to aging and morbidity are one of the reasons deaths have been concentrated among older persons around the world. In addition to having less ability to fight off a novel virus, several aspects of immune functioning also may be worse for older people. Hyperinflammation has been linked to poor outcomes with COVID-19 due to "cytokine storms," or an out-of-control immune reaction. The average number of dysregulated cytokines doubles in the age group from the $50 \mathrm{~s}$ to the $80 \mathrm{~s} .{ }^{(27)}$

In our analysis, this NLR ratio was observed to be more in cases of patients belonging to the age group of 60 and above. Our analysis was in agreement with the previously reported data; therefore, it is well understood that not only monitoring the immunological profiles but also the patient's age should be taken into consideration in the case of COVID-19.

\section{Conclusion}

Our retrospective analysis of clinical data revealed that such parameters as CRP, D-dimer, and NLR should be taken into consideration to predict severe COVID-19-related complications. The data obtained indicate that patients over age 60 are especially vulnerable to severe COVID- 19 .

\section{Competing Interests}

The authors declare that they have no competing interests. 


\section{References}

1. Sanyaolu A, Okorie C, Marinkovic A, Patidar R, Younis K, Desai P, Hosein Z, Padda I, Mangat J, Altaf M. Comorbidity and its Impact on Patients with COVID-19. SN Compr Clin Med. 2020 Jun 25:1-8. doi: 10.1007/ s42399-020-00363-4.

2. Hoffmann M, Kleine-Weber H, Schroeder S, Krüger N, Herrler T, Erichsen S, Schiergens TS, Herrler G, Wu NH, Nitsche A, Müller MA, Drosten C, Pöhlmann S. SARS-CoV-2 Cell Entry Depends on ACE2 and TMPRSS2 and Is Blocked by a Clinically Proven Protease Inhibitor. Cell. 2020 Apr 16;181(2):271-280.e8. doi: 10.1016/j. cell.2020.02.052.

3. Walls AC, Park YJ, Tortorici MA, Wall A, McGuire AT, Veesler D. Structure, Function, and Antigenicity of the SARS-CoV-2 Spike Glycoprotein. Cell. 2020 Apr 16;181(2):281-292.e6. doi: 10.1016/j.cell.2020.02.058. Epub 2020 Mar 9. Erratum in: Cell. 2020 Dec 10;183(6):1735.

4. Sungnak W, Huang N, Bécavin C, Berg M, Queen R, Litvinukova M, Talavera-López C, Maatz H, Reichart D, Sampaziotis F, Worlock KB, Yoshida M, Barnes JL; HCA Lung Biological Network. SARS-CoV-2 entry factors are highly expressed in nasal epithelial cells together with innate immune genes. Nat Med. 2020 May;26(5):681-687. doi: 10.1038/s41591-020-0868-6.

5. Siddiqi HK, Mehra MR. COVID-19 illness in native and immunosuppressed states: A clinical-therapeutic staging proposal. J Heart Lung Transplant. 2020 May;39(5):405407. doi: 10.1016/j.healun.2020.03.012.

6. Shang Y, Pan C, Yang X, Zhong M, Shang X, Wu Z, Yu Z, Zhang W, Zhong Q, Zheng X, Sang L, Jiang L, Zhang J, Xiong W, Liu J, Chen D. Management of critically ill patients with COVID-19 in ICU: statement from frontline intensive care experts in Wuhan, China. Ann Intensive Care. 2020 Jun 6;10(1):73. doi: 10.1186/s13613-02000689-1.

7. Shah VK, Firmal P, Alam A, Ganguly D, Chattopadhyay S. Overview of Immune Response During SARS-CoV-2 Infection: Lessons From the Past. Front Immunol. 2020 Aug 7;11:1949. doi: 10.3389/fimmu.2020.01949.

8. Merad M, Martin JC. Pathological inflammation in patients with COVID-19: a key role for monocytes and macrophages. Nat Rev Immunol. 2020 Jun;20(6):355-362. doi: 10.1038/s41577-020-0331-4.

9. Huertas A, Montani D, Savale L, Pichon J, Tu L, Parent F, Guignabert C, Humbert M. Endothelial cell dysfunction: a major player in SARS-CoV-2 infection (COVID-19)? Eur Respir J. 2020 Jul 30;56(1):2001634. doi: 10.1183/13993003.01634-2020.

10. McFadyen JD, Stevens H, Peter K. The Emerging Threat of (Micro)Thrombosis in COVID-19 and Its Therapeutic Implications. Circ Res. 2020 Jul 31;127(4):571-587. doi: 10.1161/CIRCRESAHA.120.317447.

11. Sakr Y, Giovini M, Leone M, Pizzilli G, Kortgen A, Bauer M, Tonetti T, Duclos G, Zieleskiewicz L, Buschbeck $\mathrm{S}$, Ranieri VM, Antonucci E. Pulmonary embolism in patients with coronavirus disease-2019 (COVID-19) pneumonia: a narrative review. Ann Intensive Care. 2020 Sep 16;10:124. doi: 10.1186/s13613-020-00741-0.

12. Wu J, Liu J, Zhao X, Liu C, Wang W, Wang D, $\mathrm{Xu}$ W, Zhang C, Yu J, Jiang B, Cao H, Li L. Clinical Characteristics of Imported Cases of Coronavirus Disease 2019 (COVID-19) in Jiangsu Province: A Multicenter Descriptive Study. Clin Infect Dis. 2020 Jul 28;71(15):706712. doi: 10.1093/cid/ciaa199.

13. Zhou F, Yu T, Du R, Fan G, Liu Y, Liu Z, Xiang J, Wang Y, Song B, Gu X, Guan L, Wei Y, Li H, Wu X, Xu J, Tu S, Zhang Y, Chen H, Cao B. Clinical course and risk factors for mortality of adult inpatients with COVID-19 in Wuhan, China: a retrospective cohort study. Lancet. 2020 Mar 28;395(10229):1054-1062. doi: 10.1016/S01406736(20)30566-3.

14. Yao Y, Cao J, Wang Q, Shi Q, Liu K, Luo Z, Chen X, Chen S, Yu K, Huang Z, Hu B. D-dimer as a biomarker for disease severity and mortality in COVID-19 patients: a case control study. J Intensive Care. 2020 Jul 10;8:49. doi: 10.1186/s40560-020-00466-z.

15. Stringer D, Braude P, Myint PK, Evans L, Collins JT, Verduri A, Quinn TJ, Vilches-Moraga A, Stechman MJ, Pearce L, Moug S, McCarthy K, Hewitt J, Carter B; COPE Study Collaborators. The role of C-reactive protein as a prognostic marker in COVID-19. Int J Epidemiol. 2021 May 17;50(2):420-429. doi: 10.1093/ije/dyab012.

16. Xu Z, Shi L, Wang Y, Zhang J, Huang L, Zhang C, Liu S, Zhao P, Liu H, Zhu L, Tai Y, Bai C, Gao T, Song J, Xia P, Dong J, Zhao J, Wang FS. Pathological findings of COVID-19 associated with acute respiratory distress syndrome. Lancet Respir Med. 2020 Apr;8(4):420-422. doi: 10.1016/S2213-2600(20)30076-X.

17. Channappanavar R, Perlman S. Pathogenic human coronavirus infections: causes and consequences of cytokine storm and immunopathology. Semin Immunopathol. 2017 Jul;39(5):529-539. doi: 10.1007/s00281-017-0629-x.

18. Alipoor SD, Jamaati H, Tabarsi P, Mortaz E. Immunopathogenesis of Pneumonia in COVID-19. Tanaffos. 2020 Nov;19(2):79-82.

19. Borges L, Pithon-Curi TC, Curi R, Hatanaka E. COVID-19 and Neutrophils: The Relationship between Hyperinflammation and Neutrophil Extracellular Traps. Mediators Inflamm. 2020 Dec 2;2020:8829674. doi: $10.1155 / 2020 / 8829674$.

20. Rao GHR. Twindemic of Coronavirus Disease (COVID-19) and Cardiometabolic Diseases. International Journal of Biomedicine. 2021;11(2):111-122. doi. org/10.21103/Article11(2)_RAI

21. Rao GHR. Coronavirus (COVID-19), Comorbidities, and Acute Vascular Events; Guest Editorial. ECCMC EC Clinical Case Reports. 2020; 3.6:87-91.

22. Bg S, Gosavi S, Ananda Rao A, Shastry S, Raj SC, Sharma A, Suresh A, Noubade R. Neutrophil-toLymphocyte, Lymphocyte-to-Monocyte, and Plateletto-Lymphocyte Ratios: Prognostic Significance in COVID-19. Cureus. 2021 Jan 11;13(1):e12622. doi: 10.7759 /cureus. 12622 .

23. Guan WJ, Ni ZY, Hu Y, Liang WH, Ou CQ, He JX, Liu L, Shan H, Lei CL, Hui DSC, Du B, Li LJ, Zeng G, 
Yuen KY, Chen RC, Tang CL, Wang T, Chen PY, Xiang J, Li SY, Wang JL, Liang ZJ, Peng YX, Wei L, Liu Y, Hu YH, Peng P, Wang JM, Liu JY, Chen Z, Li G, Zheng ZJ, Qiu SQ, Luo J, Ye CJ, Zhu SY, Zhong NS; China Medical Treatment Expert Group for Covid-19. Clinical Characteristics of Coronavirus Disease 2019 in China. N Engl J Med. 2020 Apr 30;382(18):1708-1720. doi: 10.1056/NEJMoa2002032.

24. Piazza G, Morrow DA. Diagnosis, Management, and Pathophysiology of Arterial and Venous Thrombosis in COVID-19. JAMA. 2020 Dec 22;324(24):2548-2549. doi: 10.1001/jama.2020.23422.

25. Nalbant A, Kaya T, Varim C, Yaylaci S, Tamer A, Cinemre H. Can the neutrophil/lymphocyte ratio
(NLR) have a role in the diagnosis of coronavirus 2019 disease (COVID-19)? Rev Assoc Med Bras (1992). 2020 Jun;66(6):746-751. doi: 10.1590/1806-9282.66.6.746.

26. Seyit M, Avci E, Nar R, Senol H, Yilmaz A, Ozen M, Oskay A, Aybek H. Neutrophil to lymphocyte ratio, lymphocyte to monocyte ratio and platelet to lymphocyte ratio to predict the severity of COVID-19. Am J Emerg Med. 2021 Feb;40:110-114. doi: 10.1016/j. ajem.2020.11.058.

27. Crimmins EM. Age-Related Vulnerability to Coronavirus Disease 2019 (COVID-19): Biological, Contextual, and Policy-Related Factors. Public Policy Aging Rep. 2020;30(4):142-146. doi: 10.1093/ppar/ praa023. 